\title{
FINITE ELEMENT APPROXIMATION OF THE LINEAR STOCHASTIC WAVE EQUATION WITH ADDITIVE NOISE *
}

\author{
MIHÁLY KOVÁCS ${ }^{\dagger}$, STIG LARSSON ${ }^{\ddagger}$, AND FARDIN SAEDPANAH ${ }^{\S}$
}

\begin{abstract}
Semidiscrete finite element approximation of the linear stochastic wave equation with additive noise is studied in a semigroup framework. Optimal error estimates for the deterministic problem are obtained under minimal regularity assumptions. These are used to prove strong convergence estimates for the stochastic problem. The theory presented here applies to multi-dimensional domains and spatially correlated noise. Numerical examples illustrate the theory.
\end{abstract}

Key words. finite element method, stochastic wave equation, additive noise, Wiener process, stability, a priori error estimate, strong convergence

AMS subject classifications. 65M60, 60H15, 60H35, 65C30

1. Introduction. We study finite element approximation of the linear stochastic wave equation driven by additive noise,

$$
\begin{array}{ll}
\mathrm{d} \dot{u}-\Delta u \mathrm{~d} t=\mathrm{d} W & \text { in } \mathcal{D} \times(0, \infty), \\
u=0 & \text { in } \partial \mathcal{D} \times(0, \infty), \\
u(\cdot, 0)=u_{0}, \quad \dot{u}(\cdot, 0)=v_{0} & \text { in } \mathcal{D},
\end{array}
$$

where $\mathcal{D} \subset \mathbb{R}^{d}, d=1,2,3$, is a bounded convex polygonal domain with boundary $\partial \mathcal{D}$, and $\{W(t)\}_{t \geq 0}$ is an $L_{2}(\mathcal{D})$-valued Wiener process on a filtered probability space $\left(\Omega, \mathcal{F}, \mathbf{P},\left\{\mathcal{F}_{t}\right\}_{t \geq 0}\right)$ with respect to the normal filtration $\left\{\mathcal{F}_{t}\right\}_{t \geq 0}$. We let $u_{0}$, v $v_{0}$ be $\mathcal{F}_{0}$-measurable random variables.

For introduction to the stochastic wave equation and its applications we refer to [1, 4], [5, 19] and the references therein.

The stochastic heat equation and its numerical approximation has been extensively researched in the literature, see, for example, [4, [8, [9, 11, [19, 21], 22. The numerical analysis of the stochastic wave equation is less studied, see [12, [14, [16], 20]. In particular, these works do not deal with multiple dimensions or correlated noise. This is the purpose of the present work.

We use the semigroup framework of $[5]$ in which the weak solution of 1.1$]$ is represented as a stochastic convolution

$$
u(t)=\int_{0}^{t} \Lambda^{-1 / 2} \sin \left((t-s) \Lambda^{1 / 2}\right) \mathrm{d} W(s),
$$

where, for simplicity, we have set the initial values $u_{0}=v_{0}=0$. Here $\Lambda=-\Delta$ with $D(\Lambda)=H^{2}(\mathcal{D}) \cap H_{0}^{1}(\mathcal{D})$, and $v(t)=\Lambda^{-1 / 2} \sin \left(t \Lambda^{1 / 2}\right) f$ denotes the solution of

$$
\begin{aligned}
& \ddot{v}+\Lambda v=0, \quad t>0, \\
& v(0)=0, \quad \dot{v}(0)=f .
\end{aligned}
$$

${ }^{*}$ This work was supported by the Swedish Research Council (VR) and the Swedish Foundation for Strategic Research (SSF) through GMMC, the Gothenburg Mathematical Modelling Centre.

$\dagger$ Department of Mathematics and Statistics, University of Otago, P. O. Box 56, Dunedin, New Zealand(mkovacs@maths.otago.ac.nz).

${ }^{\ddagger}$ Department of Mathematical Sciences, Chalmers University of Technology and University of Gothenburg, SE-412 96 Göteborg, Sweden(stig@chalmers.se).

$\S$ Department of Mathematical Sciences, Chalmers University of Technology and University of Gothenburg, SE-412 96 Göteborg, Sweden(fardin@chalmers.se). 
We show that, if $Q$ is the covariance operator of $W$, and if

$$
\left\|\Lambda^{(\beta-1) / 2} Q^{1 / 2}\right\|_{\mathrm{HS}}<\infty,
$$

for some $\beta \geq 0$, then we have spatial regularity of order $\beta$,

$$
\left(\mathbf{E}\left(\|u(t)\|_{\dot{H}^{\beta}}^{2}\right)\right)^{1 / 2} \leq C t^{1 / 2}\left\|\Lambda^{(\beta-1) / 2} Q^{1 / 2}\right\|_{\mathrm{HS}},
$$

where $\dot{H}^{\beta}=D\left(\Lambda^{\beta / 2}\right)$ and $\|\cdot\|_{\text {HS }}$ denotes the Hilbert-Schmidt norm. In particular, if $\operatorname{Tr}(Q)=\left\|Q^{1 / 2}\right\|_{\mathrm{HS}}^{2}<\infty$ (spatially correlated noise), then we may take $\beta=1$. On the other hand if $Q=I$ (uncorrelated noise), then $\beta<1-d / 2$, that is, $\beta<1 / 2, d=1$. See Section 3 for details.

We discretize 1.1 in the spatial variables with a standard piecewise linear finite element method, and we show strong convergence estimates in various norms. For example,

$$
\left(\mathbf{E}\left(\left\|u_{h}(t)-u(t)\right\|^{2}\right)\right)^{1 / 2} \leq C(t) h^{\frac{2}{3} \beta}\left\|\Lambda^{(\beta-1) / 2} Q^{1 / 2}\right\|_{\mathrm{HS}}, \quad \beta \in[0,3],
$$

where again $u_{0}=v_{0}=0$ and $u_{h}(t)$ is the approximate solution with maximal meshsize $h$, see Theorem 5.1

As a comparison, we recall from 21] that for the stochastic heat equation we have

$$
\begin{gathered}
\left(\mathbf{E}\left(\|u(t)\|_{\dot{H}^{\beta}}^{2}\right)\right)^{1 / 2} \leq C\left\|\Lambda^{(\beta-1) / 2} Q^{1 / 2}\right\|_{\mathrm{HS}}, \quad \beta \geq 0, \\
\left(\mathbf{E}\left(\left\|u_{h}(t)-u(t)\right\|^{2}\right)\right)^{1 / 2} \leq C h^{\beta}\left\|\Lambda^{(\beta-1) / 2} Q^{1 / 2}\right\|_{\mathrm{HS}}, \quad \beta \in[0,2] .
\end{gathered}
$$

Here the order of regularity coincides with the order of convergence.

The main tools for the proof of (1.3) are the Itô-isometry (2.4) and error estimates for the deterministic problem 1.2 with minimal regularity assumptions,

$$
\begin{gathered}
\left\|v_{h}(t)-v(t)\right\| \leq C(t) h^{2}\|f\|_{\dot{H}^{2}}, \\
\left\|v_{h}(t)-v(t)\right\| \leq C\|f\|_{\dot{H}^{-1}}
\end{gathered}
$$

and, hence by interpolation, see Corollary 4.2 ,

$$
\left\|v_{h}(t)-v(t)\right\| \leq C(t) h^{\frac{2}{3} \beta}\|f\|_{\dot{H}^{\beta}}, \quad \beta \in[0,3] .
$$

As mentioned above, when we specialize to $Q=I, d=1$, we have $\beta<1 / 2$ and thus the order of strong convergence is $O\left(h^{\alpha}\right), \alpha<1 / 3$. This is the same order as in [14, where spatial semi-discretization of the nonlinear stochastic wave equation with a standard difference scheme of uniform meshsize $h$ is considered for $d=1$ and with space-time white noise $(Q=I)$. We note that the order of convergence is less than the order of regularity, which is $\beta<1 / 2$. However, it is known that in 1.4$],\|f\|_{\dot{H}^{2}}$ can not be replaced by $\|f\|_{\dot{H}^{2-\epsilon}}$ for any $\epsilon>0$, see [15] and Remark 4.3 below. Therefore, $O\left(h^{\alpha}\right), \alpha<1 / 3$, is the best that one can expect. This explains the discrepancy in the convergence behavior between the heat and wave equations.

In [20] the leap-frog scheme is applied to the nonlinear stochastic wave equation in the unbounded domain $\mathcal{D}=\mathbb{R}$, and a strong convergence rate $O\left(h^{1 / 2}\right)$ is proved. The 
proofs in both [14] and [20] are based on representation of the exact and approximate solutions by means of Green's functions. The difference in convergence rate between the two is explained by the fact that in $\mathbb{R}$ the Green's functions for the wave equation and the leap-frog scheme coincide at mesh points, see Remark 5.2 for more details.

In summary we may say that we extend the results of $[14$ to the finite element method in multiple dimensions and to correlated noise. But we only consider the linear equation with additive noise. We also explain the discrepancy between 14 and [20]. We plan to address the nonlinear equation $\mathrm{d} \dot{u}-\Delta u \mathrm{~d} t=f(u) \mathrm{d} t+g(u) \mathrm{d} W$ in future work. Weak convergence of the finite element method is studied in [10].

The paper is organized as follows. In Section 2 some preliminaries are provided and a rigorous meaning to the infinite dimensional Wiener process $\{W(t)\}_{t \geq 0}$ and the stochastic integral are given together with the definition of a weak solution of (1.1). Existence, uniqueness, and regularity of weak solutions are discussed in Section 3 . In Section 4 the finite element method for the deterministic problem is formulated and analyzed. The results obtained here are used in Section 5 to derive strong convergence estimates for finite element approximation of the stochastic equation (1.1). Finally, numerical experiments are presented in Section 6 in order to illustrate the theory.

2. Preliminaries. Throughout the paper we use '.' to denote the time derivative ' $\frac{\partial}{\partial t}$ ', and $C$ to denote a generic positive constant, not necessarily the same at different occurrences. We refer to [5] and [13] for more details on stochastic integration and for some concepts that we cannot explain here.

Let $\left(U,(\cdot, \cdot)_{U}\right)$ and $\left(H,(\cdot, \cdot)_{H}\right)$ be separable Hilbert spaces with corresponding norms $\|\cdot\|_{U}$ and $\|\cdot\|_{H}$. We suppress the subscripts when it causes no confusion. Let $\mathcal{L}(U, H)$ denote the space of bounded linear operators from $U$ to $H$, and $\mathcal{L}_{2}(U, H)$ the space of Hilbert-Schmidt operators, endowed with norm $\|\cdot\|_{\mathcal{L}_{2}(U, H)}$. That is, $T \in \mathcal{L}_{2}(U, H)$ if $T \in \mathcal{L}(U, H)$ and

$$
\|T\|_{\mathcal{L}_{2}(U, H)}^{2}:=\sum_{k=1}^{\infty}\left\|T e_{k}\right\|_{H}^{2}<\infty
$$

where $\left\{e_{k}\right\}_{k=1}^{\infty}$ is an arbitrary ON-basis in $U$. If $H=U$ we write $\mathcal{L}(U)=\mathcal{L}(U, U)$ and HS $=\mathcal{L}_{2}(U, U)$. It is well known that if $S \in \mathcal{L}(U)$ and $T \in \mathcal{L}_{2}(U, H)$, then $T S \in \mathcal{L}_{2}(U, H)$ and we have the norm inequality

$$
\|T S\|_{\mathcal{L}_{2}(U, H)} \leq\|T\|_{\mathcal{L}_{2}(U, H)}\|S\|_{\mathcal{L}(U)}
$$

Let $(\Omega, \mathcal{F}, \mathbf{P})$ be a probability space. We define $L_{2}(\Omega, H)$ to be the space of $H$-valued square integrable random variables with norm

$$
\|v\|_{L_{2}(\Omega, H)}=\mathbf{E}\left(\|v\|_{H}^{2}\right)^{1 / 2}=\left(\int_{\Omega}\|v(\omega)\|_{H}^{2} \mathrm{~d} \mathbf{P}(\omega)\right)^{1 / 2},
$$

where $\mathbf{E}$ stands for expected value. Let $Q \in \mathcal{L}(U)$ be a selfadjoint, positive semidefinite operator, with $\operatorname{Tr}(Q)<\infty$, where $\operatorname{Tr}(Q)$ denotes the trace of $Q$. We say that $\{W(t)\}_{t \geq 0}$ is a $U$-valued $Q$-Wiener process with respect to $\left\{\mathcal{F}_{t}\right\}_{t \geq 0}$ if

(i) $W(0)=0$

(ii) $W$ has continuous trajectories (almost surely),

(iii) $W$ has independent increments,

(iv) $W(t)-W(s), 0 \leq s \leq t$, is a $U$-valued Gaussian random variable with zero mean and covariance operator $(t-s) Q$, 
and

(v) $\{W(t)\}_{t \geq 0}$ is adapted to $\left\{\mathcal{F}_{t}\right\}_{t \geq 0}$; that is, $W(t)$ is $\mathcal{F}_{t}$ measurable for all $t \geq 0$;

(vi) the random variable $W(t)-W(s)$ is independent of $\mathcal{F}_{s}$ for all fixed $s \in[0, t]$.

It is known, see, e.g., [13, Section 2.1], that for a given $Q$-Wiener process satisfying (i)-(iv) one can always find a normal filtration $\left\{\mathcal{F}_{t}\right\}_{t \geq 0}$ so that (v)-(vi) holds. Furthermore, $W(t)$ has the orthogonal expansion

$$
W(t)=\sum_{j=1}^{\infty} \gamma_{j}^{1 / 2} \beta_{j}(t) e_{j}
$$

where $\left\{\left(\gamma_{j}, e_{j}\right)\right\}_{j=1}^{\infty}$ are the eigenpairs of $Q$ with orthonormal eigenvectors and $\left\{\beta_{j}\right\}_{j=1}^{\infty}$ is a sequence of real-valued mutually independent standard Brownian motions. We note that the series in 2.2 converges in $L_{2}(\Omega, U)$, since for $t \geq 0$, we have

$$
\begin{aligned}
\|W(t)\|_{L_{2}(\Omega, U)}^{2} & =\mathbf{E}\left(\left\|\sum_{j=1}^{\infty} \gamma_{j}^{1 / 2} e_{j} \beta_{j}(t)\right\|_{U}^{2}\right)=\sum_{j=1}^{\infty} \gamma_{j} \mathbf{E}\left(\beta_{j}(t)\right)^{2} \\
& =t \sum_{j=1}^{\infty} \gamma_{j}=t \operatorname{Tr}(Q)<\infty
\end{aligned}
$$

We only need a special case of the Itô integral where the integrand is deterministic. If a function $\Phi:[0, \infty) \rightarrow \mathcal{L}(U, H)$ is strongly measurable and

$$
\int_{0}^{t}\left\|\Phi(s) Q^{1 / 2}\right\|_{\mathrm{HS}}^{2} \mathrm{~d} s<\infty
$$

then the stochastic integral $\int_{0}^{t} \Phi(s) \mathrm{d} W(s)$ is defined and we have Itô's isometry,

$$
\left\|\int_{0}^{t} \Phi(s) \mathrm{d} W(s)\right\|_{L_{2}(\Omega, H)}^{2}=\int_{0}^{t}\left\|\Phi(s) Q^{1 / 2}\right\|_{\mathcal{L}_{2}(U, H)}^{2} \mathrm{~d} s .
$$

More generally, if $Q \in \mathcal{L}(U)$ is a selfadjoint, positive semidefinite operator with eigenpairs $\left\{\left(\gamma_{j}, e_{j}\right)\right\}_{j=1}^{\infty}$, but not trace class, that is, $\operatorname{Tr}(Q)=\infty$, then the series (2.2) does not converge in $L_{2}(\Omega, U)$. However, it converges in a suitably chosen (usually larger) Hilbert space and the stochastic integral $\int_{0}^{t} \Phi(s) \mathrm{d} W(s)$ can still be defined and the isometry (2.4) holds, as long as 2.3 is satisfied. In this case $W$ is called a cylindrical Wiener process. In particular, we may have $Q=I$ (the identity operator).

Next we consider the abstract stochastic differential equation

$$
\mathrm{d} X(t)=A X(t) \mathrm{d} t+B \mathrm{~d} W(t), \quad t>0 ; \quad X(0)=X_{0}
$$

and assume that

(a1) $A: D(A) \subset H \rightarrow H$ is the generator of a strongly continuous semigroup $\left(C_{0}\right.$-semigroup) of bounded linear operators $\{E(t)\}_{t \geq 0}$ on $H$,

(a2) $B \in \mathcal{L}(U, H)$

(a3) $X_{0}$ is an $\mathcal{F}_{0}$-measurable $H$-valued random variable.

An $H$-valued predictable process $\{X(t)\}_{t \geq 0}$ is called a weak solution of (2.5), if the trajectories of $X$ are $\mathbf{P}$-a.s. Bochner integrable and, for all $\eta \in D\left(A^{*}\right)$ and all $t \geq 0$,

$$
(X(t), \eta)=\left(X_{0}, \eta\right)+\int_{0}^{t}\left(X(s), A^{*} \eta\right) \mathrm{d} s+\int_{0}^{t}(B \mathrm{~d} W(s), \eta), \quad \text { P-a.s. }
$$


3. Abstract framework and regularity. As in the introduction, let $\Lambda=-\Delta$ be the Laplace operator with $D(\Lambda)=H^{2}(\mathcal{D}) \cap H_{0}^{1}(\mathcal{D})$ and let $U=L_{2}(\mathcal{D})$ with the usual inner product $(\cdot, \cdot)$ and norm $\|\cdot\|$. In order to describe the spatial regularity of functions we introduce the following spaces and norms. Let

$$
\dot{H}^{\alpha}:=D\left(\Lambda^{\alpha / 2}\right), \quad\|v\|_{\alpha}:=\left\|\Lambda^{\alpha / 2} v\right\|=\left(\sum_{j=1}^{\infty} \lambda_{j}^{\alpha}\left(v, \phi_{j}\right)^{2}\right)^{1 / 2}, \quad \alpha \in \mathbb{R}, v \in \dot{H}^{\alpha},
$$

where $\left\{\left(\lambda_{j}, \phi_{j}\right)\right\}_{j=1}^{\infty}$ are the eigenpairs of $\Lambda$ with orthonormal eigenvectors. Then $\dot{H}^{\alpha} \subset \dot{H}^{\beta}$ for $\alpha \geq \beta$. It is known that $\dot{H}^{0}=U, \dot{H}^{1}=H_{0}^{1}(\mathcal{D}), \dot{H}^{2}=H^{2}(\mathcal{D}) \cap H_{0}^{1}(\mathcal{D})$ with equivalent norms and that $\dot{H}^{-\beta}$ can be identified with the dual space $\left(\dot{H}^{\beta}\right)^{*}$ for $\beta>0$, see [18. We note that the inner product in $\dot{H}^{1}$ is $(\cdot, \cdot)_{1}=(\nabla \cdot, \nabla \cdot)$. We also introduce

$$
H^{\alpha}:=\dot{H}^{\alpha} \times \dot{H}^{\alpha-1}, \quad\|v\|_{\alpha}^{2}:=\left\|v_{1}\right\|_{\alpha}^{2}+\left\|v_{2}\right\|_{\alpha-1}^{2}, \quad \alpha \in \mathbb{R}
$$

and set $H=H^{0}=\dot{H}^{0} \times \dot{H}^{-1}$ with corresponding norm $\||\cdot|\|\left|=\||\cdot|\|_{0}\right.$.

Next we write (1.1) as an abstract stochastic differential equation (2.5). To this end, we put $u_{1}=u, u_{2}=\dot{u}$ and note that (1.1) is formally

$$
\mathrm{d}\left[\begin{array}{l}
u_{1} \\
u_{2}
\end{array}\right]=\left[\begin{array}{cc}
0 & I \\
-\Lambda & 0
\end{array}\right]\left[\begin{array}{l}
u_{1} \\
u_{2}
\end{array}\right] \mathrm{d} t+\left[\begin{array}{l}
0 \\
I
\end{array}\right] \mathrm{d} W .
$$

We therefore define

$$
\begin{aligned}
& A:=\left[\begin{array}{cc}
0 & I \\
-\Lambda & 0
\end{array}\right], \quad B:=\left[\begin{array}{l}
0 \\
I
\end{array}\right], \quad X:=\left[\begin{array}{l}
u_{1} \\
u_{2}
\end{array}\right], \quad X_{0}:=\left[\begin{array}{l}
u_{0} \\
v_{0}
\end{array}\right], \\
& H:=H^{0}=\dot{H}^{0} \times \dot{H}^{-1}, \quad U:=\dot{H}^{0},
\end{aligned}
$$

with

$$
D(A)=\left\{x \in H: A x=\left[\begin{array}{c}
x_{2} \\
-\Lambda x_{1}
\end{array}\right] \in H=\dot{H}^{0} \times \dot{H}^{-1}\right\}=H^{1}=\dot{H}^{1} \times \dot{H}^{0} .
$$

Here $\Lambda$ is regarded as an operator $\dot{H}^{1} \rightarrow \dot{H}^{-1}$. The operator $A$ is the generator of a strongly continuous semigroup $\left(C_{0}\right.$-semigroup $) E(t)=\mathrm{e}^{t A}$ on $H$ and

$$
E(t)=\mathrm{e}^{t A}=\left[\begin{array}{cc}
C(t) & \Lambda^{-1 / 2} S(t) \\
-\Lambda^{1 / 2} S(t) & C(t)
\end{array}\right]
$$

where $C(t)=\cos \left(t \Lambda^{1 / 2}\right)$ and $S(t)=\sin \left(t \Lambda^{1 / 2}\right)$ are the cosine and sine operators. For example, using $\left\{\left(\lambda_{j}, \phi_{j}\right)\right\}_{j=1}^{\infty}$, the orthonormal eigenpairs of $\Lambda$, we have

$$
\Lambda^{-1 / 2} S(t) v=\Lambda^{-1 / 2} \sin \left(t \Lambda^{1 / 2}\right) v=\sum_{j=1}^{\infty} \lambda_{j}^{-1 / 2} \sin \left(t \lambda_{j}^{1 / 2}\right)\left(v, \phi_{j}\right) \phi_{j} .
$$

We also note that $B \in \mathcal{L}(U, H)$ and we let $X_{0}$ be an $\mathcal{F}_{0}$-measurable $H$-valued random variable to fulfill the assumptions (a1)-(a3). We assume that $W$ is a $Q$-Wiener process or a cylindrical Wiener process on $U$. Now (1.1) is set in the form 2.5), which is given a rigorous meaning by the weak formulation (2.6). Next we consider the existence, 
uniqueness, and regularity of the weak solution. Recall that we write $\mathrm{HS}=\mathcal{L}_{2}(U, U)$ for the Hilbert-Schmidt operators on $U$.

The existence and uniqueness of the weak solution of the stochastic wave equation is discussed in [5, Example 5.8]. However, the spatial Sobolev regularity of the solution under the hybrid condition (3.3) on $\Lambda$ and $Q$ seems to be new.

THEOREM 3.1. With the above definitions and if

$$
\left\|\Lambda^{(\beta-1) / 2} Q^{1 / 2}\right\|_{\mathrm{HS}}<\infty
$$

for some $\beta \geq 0$, then 2.5. has a unique weak solution, which is given by the variation of constants formula,

$$
X(t)=E(t) X_{0}+\int_{0}^{t} E(t-s) B \mathrm{~d} W(s), \quad t \geq 0 .
$$

Moreover,

$$
\|X(t)\|_{L_{2}\left(\Omega, H^{\beta}\right)} \leq C\left(\left\|X_{0}\right\|_{L_{2}\left(\Omega, H^{\beta}\right)}+t^{1 / 2}\left\|\Lambda^{(\beta-1) / 2} Q^{1 / 2}\right\|_{\mathrm{HS}}\right), \quad t \geq 0 .
$$

Proof. To prove that (3.4) is the unique weak solution it is enough to show that, for fixed $t$,

$$
\int_{0}^{t}\left\|E(s) B Q^{1 / 2}\right\|_{\mathcal{L}_{2}(U, H)}^{2} \mathrm{~d} s<\infty
$$

see [5, Theorem 5.4]. Indeed, with $\left\{e_{k}\right\}_{k=1}^{\infty}$, an arbitrary ON-basis in $U$, and for any $\beta \geq 0$, we have

$$
\begin{aligned}
\int_{0}^{t} & \left\|E(s) B Q^{1 / 2}\right\|_{\mathcal{L}_{2}\left(U, H^{\beta}\right)}^{2} \mathrm{~d} s=\int_{0}^{t} \sum_{k=1}^{\infty}\left\|E(s) B Q^{1 / 2} e_{k} \mid\right\|_{\beta}^{2} \mathrm{~d} s \\
& =\int_{0}^{t} \sum_{k=1}^{\infty}\left\{\left\|\Lambda^{-1 / 2} S(s) Q^{1 / 2} e_{k}\right\|_{\beta}^{2}+\left\|C(s) Q^{1 / 2} e_{k}\right\|_{\beta-1}^{2}\right\} \mathrm{d} s \\
& =\int_{0}^{t}\left\{\left\|\Lambda^{(\beta-1) / 2} S(s) Q^{1 / 2}\right\|_{\mathrm{HS}}^{2}+\left\|\Lambda^{(\beta-1) / 2} C(s) Q^{1 / 2}\right\|_{\mathrm{HS}}^{2}\right\} \mathrm{d} s \\
& \leq 2 t\left\|\Lambda^{(\beta-1) / 2} Q^{1 / 2}\right\|_{\mathrm{HS}}^{2},
\end{aligned}
$$

where, for the last inequality, we used the fact that the $\Lambda$ commutes with $C(s), S(s)$ and (2.1) together with the boundedness of the cosine and the sine operators in $U$. With $\beta=0$, this implies (3.6), and therefore it implies existence and uniqueness of the weak solution. Finally, (3.5) follows from (3.4), the boundedness of $E(t)$ in $H^{\beta}$, the Itô isometry (2.4), and (3.7):

$$
\begin{aligned}
\|X(t)\|_{L_{2}\left(\Omega, H^{\beta}\right)}^{2} & \leq 2\left(\left\|E(t) X_{0}\right\|_{L_{2}\left(\Omega, H^{\beta}\right)}^{2}+\left\|\int_{0}^{t} E(t-s) B \mathrm{~d} W(s)\right\|_{L_{2}\left(\Omega, H^{\beta}\right)}^{2}\right) \\
& \leq 2\left(\left\|X_{0}\right\|_{L_{2}\left(\Omega, H^{\beta}\right)}^{2}+\int_{0}^{t}\left\|E(s) B Q^{1 / 2}\right\|_{\mathcal{L}_{2}\left(U, H^{\beta}\right)}^{2} \mathrm{~d} s\right) .
\end{aligned}
$$

This completes the proof.

Remark 3.2. The parameter $\beta$ in the condition $\left\|\Lambda^{(\beta-1) / 2} Q^{1 / 2}\right\|_{\mathrm{HS}}<\infty$ quantifies the spatial correlation of the noise. We highlight three special cases. 
1. If $Q$ is of trace class, then $\beta=1$, because $\left\|Q^{1 / 2}\right\|_{\mathrm{HS}}^{2}=\operatorname{Tr}(Q)<\infty$.

2. If $Q=I$, which corresponds to space-time white noise, then $\left\|\Lambda^{(\beta-1) / 2}\right\|_{\mathrm{HS}}<$ $\infty$ if and only if $d=1$ and $\beta<1 / 2$. Indeed, the eigenvalues of $\Lambda$ behave asymptotically like $\lambda_{j} \approx j^{2 / d}$, so that

$$
\left\|\Lambda^{(\beta-1) / 2}\right\|_{\mathrm{HS}}^{2}=\sum_{j=1}^{\infty} \lambda_{j}^{\beta-1} \approx \sum_{j=1}^{\infty} j^{2(\beta-1) / d},
$$

and the series converges if and only if $\beta<1-d / 2$, that is, $d=1, \beta<1 / 2$.

3. Similarly, if $Q=\Lambda^{-s}, s>0$, then $\beta<1+s-d / 2$.

Thus, in order to have a positive order of regularity in multiple dimensions $(d>1)$ we need correlated noise.

4. The finite element method for the deterministic problem. We need error estimates with minimal regularity requirement for the approximation of the deterministic wave equation. These are not readily available in the literature, see Remark 4.5 below, and we therefore provide a complete proof in this section.

We first study the spatially semidiscrete finite element method for the deterministic non-homogeneous linear wave equation,

$$
\begin{aligned}
& \ddot{u}-\Delta u=f \quad \text { in } \mathcal{D} \times(0, \infty), \\
& u=0 \quad \text { on } \partial \mathcal{D} \times(0, \infty) \\
& u(\cdot, 0)=u_{0}, \quad \dot{u}(\cdot, 0)=v_{0} \quad \text { in } \mathcal{D},
\end{aligned}
$$

where $\mathcal{D} \in \mathbb{R}^{d}, d=1,2,3$, is a bounded convex polygonal domain with boundary $\partial \mathcal{D}$. We then specialize to the homogeneous equation and derive estimates in a form which is suitable for the proof of strong convergence estimates for the finite element approximation of the stochastic equation.

4.1. Error estimates for the non-homogeneous problem. Let $\left\{\mathcal{T}_{h}\right\}$ be a regular family of triangulations of $\mathcal{D}$ with $h_{K}=\operatorname{diam}(K), h=\max _{K \in \mathcal{T}_{h}} h_{K}$, and denote by $V_{h}$ the space of piecewise linear continuous functions with respect to $\mathcal{T}_{h}$ which vanish on $\partial \mathcal{D}$. Hence, $V_{h} \subset H_{0}^{1}(\mathcal{D})=\dot{H}^{1}$.

The assumption that $\mathcal{D}$ is convex and polygonal guarantees that the triangulations can be exacly fitted to $\partial \mathcal{D}$ and that we have the elliptic regularity $\|v\|_{H^{2}(\mathcal{D})} \leq C\|\Lambda v\|$ for $v \in D(\Lambda)$. We can now quote basic results from the theory of finite elements. We use the norms $\|\cdot\|_{s}=\|\cdot\|_{\dot{H}^{s}}$.

For the orthogonal projectors $\mathcal{P}_{h}: \dot{H}^{0} \rightarrow V_{h}, \mathcal{R}_{h}: \dot{H}^{1} \rightarrow V_{h}$ defined by

$$
\left(\mathcal{P}_{h} v, \chi\right)=(v, \chi), \quad\left(\nabla \mathcal{R}_{h} v, \nabla \chi\right)=(\nabla v, \nabla \chi), \quad \forall \chi \in V_{h},
$$

we have the following error estimates:

$$
\begin{gathered}
\left\|\left(\mathcal{R}_{h}-I\right) v\right\|_{r} \leq C h^{s-r}\|v\|_{s}, \quad r=0,1, s=1,2, \quad v \in \dot{H}^{s}, \\
\left\|\left(\mathcal{P}_{h}-I\right) v\right\|_{r} \leq C h^{s-r}\|v\|_{s}, \quad r=-1,0, s=1,2, \quad v \in \dot{H}^{s} .
\end{gathered}
$$

We define a discrete variant of the norm $\|\cdot\|_{\alpha}$ :

$$
\left\|v_{h}\right\|_{h, \alpha}=\left\|\Lambda_{h}^{\alpha / 2} v_{h}\right\|, \quad v_{h} \in V_{h}, \alpha \in \mathbb{R},
$$

where $\Lambda_{h}: V_{h} \rightarrow V_{h}$ is the discrete Laplace operator defined by

$$
\left(\Lambda_{h} v_{h}, \chi\right)=\left(\nabla v_{h}, \nabla \chi\right), \quad \forall \chi \in V_{h} .
$$


It is clear that $\left\|v_{h}\right\|_{1, h}=\left\|\nabla v_{h}\right\|=\left\|v_{h}\right\|_{1}$ and

$$
\left\|\mathcal{P}_{h} f\right\|_{-1, h} \leq\|f\|_{-1}, \quad f \in \dot{H}^{-1}
$$

follows from the calculation

$$
\begin{aligned}
\left\|\Lambda_{h}^{-\frac{1}{2}} \mathcal{P}_{h} f\right\| & =\sup _{v_{h} \in V_{h}} \frac{\left|\left(\Lambda_{h}^{-\frac{1}{2}} \mathcal{P}_{h} f, v_{h}\right)\right|}{\left\|v_{h}\right\|}=\sup _{v_{h} \in V_{h}} \frac{\left|\left(f, \Lambda_{h}^{-\frac{1}{2}} v_{h}\right)\right|}{\left\|v_{h}\right\|} \\
& =\sup _{w_{h} \in V_{h}} \frac{\left|\left(f, w_{h}\right)\right|}{\left\|\Lambda_{h}^{\frac{1}{2}} w_{h}\right\|}=\sup _{w_{h} \in V_{h}} \frac{\left|\left(f, w_{h}\right)\right|}{\left\|w_{h}\right\|_{1}} \\
& \leq \sup _{w \in \dot{H}^{1}} \frac{|(f, w)|}{\|w\|_{1}}=\|f\|_{-1} .
\end{aligned}
$$
that

With $u_{1}=u, u_{2}=\dot{u}$, the weak form of (4.1) reads: find $u_{1}(t), u_{2}(t) \in \dot{H}^{1}$, such

$$
\begin{aligned}
& \left(\nabla \dot{u}_{1}(t), \nabla v_{1}\right)-\left(\nabla u_{2}(t), \nabla v_{1}\right)=0, \\
& \left(\dot{u}_{2}(t), v_{2}\right)+\left(\nabla u_{1}(t), \nabla v_{2}\right)=\left(f(t), v_{2}\right), \quad \forall v_{1}, v_{2} \in \dot{H}^{1}, t>0, \\
& u_{1}(0)=u_{0}, u_{2}(0)=v_{0} .
\end{aligned}
$$

The semidiscrete analogue of 4.5 is then to find $u_{h, 1}(t), u_{h, 2}(t) \in V_{h}$ such that

$$
\begin{aligned}
& \left(\nabla \dot{u}_{h, 1}(t), \nabla \chi_{1}\right)-\left(\nabla u_{h, 2}(t), \nabla \chi_{1}\right)=0, \\
& \left(\dot{u}_{h, 2}(t), \chi_{2}\right)+\left(\nabla u_{h, 1}(t), \nabla \chi_{2}\right)=\left(f(t), \chi_{2}\right), \quad \forall \chi_{1}, \chi_{2} \in V_{h}, t>0, \\
& u_{h, 1}(0)=u_{h, 0}, \quad u_{h, 1}(0)=v_{h, 0},
\end{aligned}
$$

with initial values $u_{h, 0}, v_{h, 0} \in V_{h}$.

In our error analysis we will use the stability of the slightly more general problem of finding $u_{h, 1}(t), u_{h, 2}(t) \in V_{h}$ such that

$$
\begin{aligned}
& \left(\nabla \dot{u}_{h, 1}(t), \nabla \chi_{1}\right)-\left(\nabla u_{h, 2}(t), \nabla \chi_{1}\right)=\left(\nabla f_{1}(t), \nabla \chi_{1}\right), \quad \forall \chi_{1}, \chi_{2} \in V_{h}, t>0, \\
& \left(\dot{u}_{h, 2}(t), \chi_{2}\right)+\left(\nabla u_{h, 1}(t), \nabla \chi_{2}\right)=\left(f_{2}(t), \chi_{2}\right), \\
& u_{h, 1}(0)=u_{h, 0}, \quad u_{h, 1}(0)=v_{h, 0},
\end{aligned}
$$

We set $\chi_{i}=\Lambda_{h}^{\alpha} u_{h, i}, i=1,2, \alpha \in \mathbb{R}$, in 4.7) and conclude in a standard way that

$$
\begin{aligned}
\left\|u_{h, 1}(t)\right\|_{h, \alpha+1}+ & \left\|u_{h, 2}(t)\right\|_{h, \alpha} \leq C\left\{\left\|u_{h, 0}\right\|_{h, \alpha+1}+\left\|v_{h, 0}\right\|_{h, \alpha}\right. \\
& \left.+\int_{0}^{t}\left\|\mathcal{R}_{h} f_{1}(s)\right\|_{h, \alpha+1} \mathrm{~d} s+\int_{0}^{t}\left\|\mathcal{P}_{h} f_{2}(s)\right\|_{h, \alpha} \mathrm{d} s\right\} .
\end{aligned}
$$

In the next theorem we obtain optimal order error estimates in $L_{\infty}\left([0, \infty), \dot{H}^{s}\right)$ with $s=0,1$ for $u_{h, 1}$ and $s=0$ for $u_{h, 2}$. The regularity requirement is minimal, see Remark 4.3.

THEOREM 4.1. Let $u_{1}, u_{2}$ and $u_{h, 1}, u_{h, 2}$ be the solutions of 4.5 and (4.6), respectively, and set $e_{i}:=u_{h, i}-u_{i}, i=1,2$. Then, for $t \geq 0$, we have

$$
\begin{aligned}
\left\|e_{1}(t)\right\|_{1} \leq & C\left\{\left\|u_{h, 0}-\mathcal{R}_{h} u_{0}\right\|_{1}+\left\|v_{h, 0}-\mathcal{R}_{h} v_{0}\right\|\right\} \\
& +C h\left\{\left\|u_{1}(t)\right\|_{2}+\int_{0}^{t}\left\|\dot{u}_{2}(s)\right\|_{1} \mathrm{~d} s\right\}
\end{aligned}
$$




$$
\begin{aligned}
\left\|e_{2}(t)\right\| \leq & C\left\{\left\|u_{h, 0}-\mathcal{R}_{h} u_{0}\right\|_{1}+\left\|v_{h, 0}-\mathcal{R}_{h} v_{0}\right\|\right\} \\
& +C h^{2}\left\{\left\|u_{2}(t)\right\|_{2}+\int_{0}^{t}\left\|\dot{u}_{2}(s)\right\|_{2} \mathrm{~d} s\right\}, \\
\left\|e_{1}(t)\right\| \leq & C\left\{\left\|u_{h, 0}-\mathcal{R}_{h} u_{0}\right\|+\left\|v_{h, 0}-\mathcal{P}_{h} v_{0}\right\|_{-1}\right\} \\
& +C h^{2}\left\{\left\|u_{1}(t)\right\|_{2}+\int_{0}^{t}\left\|u_{2}(s)\right\|_{2} \mathrm{~d} s\right\} .
\end{aligned}
$$

Proof. We set

$$
e_{i}=\theta_{i}+\rho_{i}=\left(u_{h, i}-\pi_{i} u\right)+\left(\pi_{i} u_{i}-u_{i}\right), \quad i=1,2,
$$

where $\pi_{i}$ will be chosen as $\mathcal{R}_{h}$ or $\mathcal{P}_{h}$. By subtraction of 4.5 and 4.6 , recalling $V_{h} \subset \dot{H}^{1}$, we obtain

$$
\begin{aligned}
& \left(\nabla \dot{e}_{1}(t), \nabla \chi_{1}\right)-\left(\nabla e_{2}(t), \nabla \chi_{1}\right)=0 \\
& \left(\dot{e}_{2}(t), \chi_{2}\right)+\left(\nabla e_{1}(t), \nabla \chi_{2}\right)=0, \quad \forall \chi_{1}, \chi_{2} \in V_{h}, t>0 .
\end{aligned}
$$

Hence,

$$
\begin{aligned}
\left(\nabla \dot{\theta}_{1}, \nabla \chi_{1}\right)-\left(\nabla \theta_{2}, \nabla \chi_{1}\right) & =-\left(\nabla \dot{\rho}_{1}, \nabla \chi_{1}\right)+\left(\nabla \rho_{2}, \nabla \chi_{1}\right), \quad \forall \chi_{1}, \chi_{2} \in V_{h}, t>0 . \\
\left(\dot{\theta}_{2}, \chi_{2}\right)+\left(\nabla \theta_{1}, \nabla \chi_{2}\right) & =-\left(\dot{\rho}_{2}, \chi_{2}\right)-\left(\nabla \rho_{1}, \nabla \chi_{2}\right),
\end{aligned}
$$

First, in order to prove the error estimates 4.9 and 4.10 , we set

$$
\theta_{i}=u_{h, i}-\mathcal{R}_{h} u_{i}, \quad \rho_{i}=\left(\mathcal{R}_{h}-I\right) u_{i}, \quad i=1,2
$$

By the definitions of the operators $\mathcal{R}_{h}, \mathcal{P}_{h}$, we have

$$
\begin{aligned}
\left(\nabla \dot{\theta}_{1}, \nabla \chi_{1}\right)-\left(\nabla \theta_{2}, \nabla \chi_{1}\right) & =0, \\
\left(\dot{\theta}_{2}, \chi_{2}\right)+\left(\nabla \theta_{1}, \nabla \chi_{2}\right) & =-\left(\dot{\rho}_{2}, \chi_{2}\right), \quad \forall \chi_{1}, \chi_{2} \in V_{h}, t>0,
\end{aligned}
$$

that is, $\theta_{1}, \theta_{2}$ satisfy 4.7 with $f_{1}=0, f_{2}=-\dot{\rho}_{2}$. Therefore, by the stability inequality 4.8 with $\alpha=0$, we obtain

$$
\left\|\theta_{1}(t)\right\|_{h, 1}+\left\|\theta_{2}(t)\right\|_{h, 0} \leq C\left\{\left\|\theta_{1}(0)\right\|_{h, 1}+\left\|\theta_{2}(0)\right\|_{h, 0}+\int_{0}^{t}\left\|\mathcal{P}_{h} \dot{\rho}_{2}(s)\right\|_{h, 0} \mathrm{~d} s\right\}
$$

Recalling (4.12 and that $\left\|v_{h}\right\|_{h, 0}=\left\|v_{h}\right\|$ and $\left\|v_{h}\right\|_{h, 1}=\left\|v_{h}\right\|_{1}, v_{h} \in V_{h}$, we have

$$
\begin{aligned}
\left\|e_{1}(t)\right\|_{1} \leq & C\left\{\left\|u_{h, 0}-\mathcal{R}_{h} u_{0}\right\|_{1}+\left\|v_{h, 0}-\mathcal{R}_{h} v_{0}\right\|\right. \\
& \left.+\int_{0}^{t}\left\|\left(\mathcal{R}_{h}-I\right) \dot{u}_{2}(s)\right\| \mathrm{d} s+\left\|\left(\mathcal{R}_{h}-I\right) u_{1}(t)\right\|_{1}\right\} \\
\left\|e_{2}(t)\right\| \leq & C\left\{\left\|u_{h, 0}-\mathcal{R}_{h} u_{0}\right\|_{1}+\left\|v_{h, 0}-\mathcal{R}_{h} v_{0}\right\|\right. \\
& \left.+\int_{0}^{t}\left\|\left(\mathcal{R}_{h}-I\right) \dot{u}_{2}(s)\right\| \mathrm{d} s+\left\|\left(\mathcal{R}_{h}-I\right) u_{2}(t)\right\|\right\} .
\end{aligned}
$$


Using 4.2 we conclude (4.9) and 4.10).

Finally, to prove the error estimate 4.11) we alter the choice of $\pi_{i}$ in 4.12,

$$
\begin{array}{ll}
\theta_{1}=u_{h, 1}-\mathcal{R}_{h} u_{1}, & \rho_{1}=\left(\mathcal{R}_{h}-I\right) u_{1}, \\
\theta_{2}=u_{h, 2}-\mathcal{P}_{h} u_{2}, & \rho_{2}=\left(\mathcal{P}_{h}-I\right) u_{2} .
\end{array}
$$

Then, similarly to the previous case,

$$
\begin{aligned}
\left(\nabla \dot{\theta}_{1}, \nabla \chi_{1}\right)-\left(\nabla \theta_{2}, \nabla \chi_{1}\right) & =\left(\nabla \rho_{2}, \nabla \chi_{1}\right), \quad \forall \chi_{1}, \chi_{2} \in V_{h}, t>0, \\
\left(\dot{\theta}_{2}, \chi_{2}\right)+\left(\nabla \theta_{1}, \nabla \chi_{2}\right) & =0,
\end{aligned}
$$

that is, $\theta_{1}, \theta_{2}$ satisfy (4.7) with $f_{1}=\rho_{2}, f_{2}=0$. Therefore, by the stability inequality 4.8 with $\alpha=-1$, we obtain

$$
\left\|\theta_{1}(t)\right\|_{h, 0}+\left\|\theta_{2}(t)\right\|_{h,-1} \leq C\left\{\left\|\theta_{1}(0)\right\|_{h, 0}+\left\|\theta_{2}(0)\right\|_{h,-1}+\int_{0}^{t}\left\|\mathcal{R}_{h} \rho_{2}(s)\right\|_{h, 0} \mathrm{~d} s\right\} .
$$

Using (4.4, 4.12, and

$$
\left\|\mathcal{R}_{h} \rho_{2}\right\|=\left\|\mathcal{P}_{h}\left(I-\mathcal{R}_{h}\right) u_{2}\right\| \leq\left\|\left(\mathcal{R}_{h}-I\right) u_{2}\right\|
$$

we have

$$
\begin{array}{r}
\left\|e_{1}(t)\right\| \leq C\left\{\left\|u_{h, 0}-\mathcal{R}_{h} u_{0}\right\|+\left\|v_{h, 0}-\mathcal{P}_{h} v_{0}\right\|_{-1}\right. \\
\left.+\int_{0}^{t}\left\|\left(\mathcal{R}_{h}-I\right) u_{2}(s)\right\| \mathrm{d} s+\left\|\left(\mathcal{R}_{h}-I\right) u_{1}(t)\right\|\right\} .
\end{array}
$$

In view of 4.2 this proves 4.11.

4.2. Error estimates for the homogeneous problem. Here we specialize to the homogeneous problem

$$
\begin{aligned}
& \ddot{u}(t)+\Lambda u(t)=0, \quad t>0, \\
& u(0)=u_{0}, \dot{u}(0)=v_{0},
\end{aligned}
$$

and express the error estimates in terms of the initial values. Differentiating the equation $r$ times with respect to $t$, we obtain in a standard way

$$
\left\|\mathrm{D}_{t}^{r} \dot{u}(t)\right\|_{\alpha}^{2}+\left\|\mathrm{D}_{t}^{r} u(t)\right\|_{\alpha+1}^{2}=\left\|v_{0}^{r}\right\|_{\alpha}^{2}+\left\|u_{0}^{r}\right\|_{\alpha+1}^{2} .
$$

Here, for $k=0,1, \ldots$,

$$
\begin{aligned}
& u_{0}^{r}=\Lambda^{k} u_{0}, \quad v_{0}^{r}=\Lambda^{k} v_{0}, \quad r=2 k, \\
& u_{0}^{r}=\Lambda^{k} v_{0}, \quad v_{0}^{r}=\Lambda^{k+1} u_{0}, \quad r=2 k+1 .
\end{aligned}
$$

We use the notation from Section 3 and we write 4.13 as

$$
\dot{X}(t)=A X(t), \quad t>0 ; \quad X(0)=X_{0},
$$

and we recall that the linear operator $A$ is the generator of a $C_{0}$-semigroup $E(t)=\mathrm{e}^{t A}$ given by (3.2). Therefore the solution is $X(t)=E(t) X_{0}$. The finite element problem is then to find $X_{h}(t) \in V_{h} \times V_{h}$ such that

$$
\dot{X}_{h}(t)=A_{h} X_{h}(t), \quad t>0 ; \quad X_{h}(0)=X_{h, 0},
$$


where

$$
A_{h}=\left[\begin{array}{cc}
0 & I \\
-\Lambda_{h} & 0
\end{array}\right], \quad X_{h}=\left[\begin{array}{l}
u_{h, 1} \\
u_{h, 2}
\end{array}\right], \quad X_{h, 0}=\left[\begin{array}{l}
u_{h, 0} \\
v_{h, 0}
\end{array}\right] .
$$

Similarly to 3.2 , it can be shown that $A_{h}$ generates a $C_{0}$-semigroup $E_{h}(t)$ given by

$$
E_{h}(t)=\mathrm{e}^{t A_{h}}=\left[\begin{array}{cc}
C_{h}(t) & \Lambda_{h}^{-1 / 2} S_{h}(t) \\
-\Lambda_{h}^{1 / 2} S_{h}(t) & C_{h}(t)
\end{array}\right]
$$

with

$$
C_{h}(t)=\cos \left(t \Lambda_{h}^{1 / 2}\right), \quad S_{h}(t)=\sin \left(t \Lambda_{h}^{1 / 2}\right) .
$$

For example, similarly to the infinite dimensional case, using $\left\{\left(\lambda_{h, j}, \phi_{h, j}\right)\right\}_{j=1}^{N_{h}}$, the orthonormal eigenpairs of the discrete Laplacian $\Lambda_{h}$, with $N_{h}=\operatorname{dim}\left(V_{h}\right)$, we have

$$
\Lambda_{h}^{-1 / 2} \sin \left(t \Lambda_{h}^{1 / 2}\right) v_{h}=\sum_{j=1}^{N_{h}} \lambda_{h, j}^{-1 / 2} \sin \left(t \lambda_{h, j}^{1 / 2}\right)\left(v_{h}, \phi_{h, j}\right) \phi_{h, j}, \quad v_{h} \in V_{h} .
$$

We may now formulate a consequence of Theorem 4.1, which will be used to prove the strong convergence of the finite element approximation of the stochastic wave equation. Recall $\|\mid v\|_{\alpha}^{2}=\left\|v_{1}\right\|_{\alpha}^{2}+\left\|v_{2}\right\|_{\alpha-1}^{2}$ from (3.1).

Corollary 4.2. Denote $X_{0}=\left[u_{0}, v_{0}\right]^{T}$ and let

$$
\begin{aligned}
& F_{h}(t) X_{0}=\left(C_{h}(t) \mathcal{P}_{h}-C(t)\right) u_{0}+\left(\Lambda_{h}^{-1 / 2} S_{h}(t) \mathcal{P}_{h}-\Lambda^{-1 / 2} S(t)\right) v_{0}, \\
& G_{h}(t) X_{0}=\left(C_{h}(t) \mathcal{R}_{h}-C(t)\right) u_{0}+\left(\Lambda_{h}^{-1 / 2} S_{h}(t) \mathcal{P}_{h}-\Lambda^{-1 / 2} S(t)\right) v_{0}, \\
& \dot{G}_{h}(t) X_{0}=-\left(\Lambda_{h}^{1 / 2} S_{h}(t) \mathcal{R}_{h}-\Lambda^{1 / 2} S(t)\right) u_{0}+\left(C_{h}(t) \mathcal{P}_{h}-C(t)\right) v_{0} .
\end{aligned}
$$

Then we have

$$
\begin{aligned}
\left\|F_{h}(t) X_{0}\right\| & \leq C(1+t) h^{\frac{2}{3} \beta}\left\|\mid X_{0}\right\| \|_{\beta}, \quad t \geq 0, \quad \beta \in[0,3], \\
\left\|G_{h}(t) X_{0}\right\|_{1} & \leq C(1+t) h^{\frac{1}{2}(\beta-1)}\|\| X_{0} \|\left.\right|_{\beta}, \quad t \geq 0, \quad \beta \in[1,3], \\
\left\|\dot{G}_{h}(t) X_{0}\right\| & \leq C(1+t) h^{\frac{2}{3}(\beta-1)}\|\mid\| X_{0} \|_{\beta}, \quad t \geq 0, \quad \beta \in[1,4] .
\end{aligned}
$$

Note that $F_{h}$ and $G_{h}$ differ only in the choice of initial value: $u_{h, 0}=\mathcal{P}_{h} u_{0}$ and $u_{h, 0}=\mathcal{R}_{h} u_{0}$. This is necessary in order to accommodate the lowest order of initial regularity used $(\beta=0$ and $\beta=1)$.

Proof. We begin with the case $\beta=0$ of 4.18. By the stability (4.8) with $\alpha=-1$ and its analogue for the continuous equation, and 4.4, we have

$$
\begin{aligned}
\left\|F_{h}(t) X_{0}\right\| & \leq\left\|u_{h, 1}(t)\right\|+\left\|u_{1}(t)\right\| \leq C\left\{\left\|\mathcal{P}_{h} u_{0}\right\|+\left\|\mathcal{P}_{h} v_{0}\right\|_{-1, h}+\left\|u_{0}\right\|+\left\|v_{0}\right\|_{-1}\right\} \\
& \leq C\left(\left\|u_{0}\right\|+\left\|v_{0}\right\|_{-1}\right)=C\|\| X_{0} \|_{0} .
\end{aligned}
$$

For the case $\beta=3$ we use (4.11) with $u_{h, 0}=\mathcal{P}_{h} u_{0}$ and $v_{h, 0}=\mathcal{P}_{h} v_{0}$, and (4.14),

$$
\begin{aligned}
\left\|F_{h}(t) X_{0}\right\| & =\left\|e_{1}(t)\right\| \leq C\left\{\left\|\mathcal{P}_{h}\left(I-\mathcal{R}_{h}\right) u_{0}\right\|\right\}+C h^{2}\left\{\left\|u_{1}(t)\right\|_{2}+\int_{0}^{t}\left\|u_{2}(s)\right\|_{2} \mathrm{~d} s\right\} \\
& \leq C h^{2}\left\{\left\|u_{0}\right\|_{2}+\left\|v_{0}\right\|_{1}+t\left(\left\|u_{0}\right\|_{3}+\left\|v_{0}\right\|_{2}\right)\right\} \leq C(1+t) h^{2}\left\|X_{0}\right\|_{3} .
\end{aligned}
$$


The proof is then completed by interpolation between these cases.

For 4.19 we first use (4.8) with $\alpha=0$,

$$
\begin{aligned}
\left\|G_{h}(t) X_{0}\right\|_{1} & \leq\left\|u_{h, 1}(t)\right\|_{1}+\left\|u_{1}(t)\right\|_{1} \leq C\left\{\left\|\mathcal{R}_{h} u_{0}\right\|_{1}+\left\|\mathcal{P}_{h} v_{0}\right\|+\left\|u_{0}\right\|_{1}+\left\|v_{0}\right\|\right\} \\
& \leq C\left(\left\|u_{0}\right\|_{1}+\left\|v_{0}\right\|\right)=C \mid\left\|X_{0}\right\| \|_{1} .
\end{aligned}
$$

Then we use 4.9. with $u_{h, 0}=\mathcal{R}_{h} u_{0}$ and $v_{h, 0}=\mathcal{P}_{h} v_{0}$,

$$
\begin{aligned}
\left\|G_{h}(t) X_{0}\right\|_{1} & =\left\|e_{1}(t)\right\|_{1} \leq C\left\{\left\|\mathcal{P}_{h}\left(I-\mathcal{R}_{h}\right) v_{0}\right\|\right\}+C h\left\{\left\|u_{1}(t)\right\|_{2}+\int_{0}^{t}\left\|\dot{u}_{2}(s)\right\|_{1} \mathrm{~d} s\right\} \\
& \leq C h\left\{\left\|u_{0}\right\|_{2}+\left\|v_{0}\right\|_{1}+t\left(\left\|u_{0}\right\|_{3}+\left\|v_{0}\right\|_{2}\right)\right\} \leq C(1+t) h^{2}\|\| X_{0}\|\|_{3} .
\end{aligned}
$$

For 4.20 we apply 4.8 with $\alpha=0$,

$$
\begin{aligned}
\left\|\dot{G}_{h}(t) X_{0}\right\| & \leq\left\|u_{h, 2}(t)\right\|+\left\|u_{2}(t)\right\| \leq C\left\{\left\|\mathcal{R}_{h} u_{0}\right\|_{1}+\left\|\mathcal{P}_{h} v_{0}\right\|+\left\|u_{0}\right\|_{1}+\left\|v_{0}\right\|\right\} \\
& \leq C\left(\left\|u_{0}\right\|_{1}+\left\|v_{0}\right\|\right)=C\|\| X_{0} \|_{1} .
\end{aligned}
$$

Then we use 4.10 with $u_{h, 0}=\mathcal{R}_{h} u_{0}$ and $v_{h, 0}=\mathcal{P}_{h} v_{0}$,

$$
\begin{aligned}
\left\|\dot{G}_{h}(t) X_{0}\right\| & =\left\|e_{2}(t)\right\| \leq C h^{2}\left\{\left\|u_{2}(t)\right\|_{2}+\int_{0}^{t}\left\|\dot{u}_{2}(s)\right\|_{2} \mathrm{~d} s\right\} \\
& \leq C h\left\{\left\|u_{0}\right\|_{3}+\left\|v_{0}\right\|_{2}+t\left(\left\|u_{0}\right\|_{4}+\left\|v_{0}\right\|_{3}\right)\right\} \leq C(1+t) h^{2}\left\|X_{0}\right\|_{4} .
\end{aligned}
$$

This completes the proof. $\square$

Remark 4.3. The regularity assumption on $X_{0}$ in Corollary 4.2 cannot be relaxed. This means that $\left\|\mid X_{0}\right\|_{\beta}$ can not be replaced by $\left\|\mid X_{0}\right\| \|_{\beta-\epsilon}$ for any $\epsilon>0$. This is shown in the lemma below for the periodic problem

$$
\begin{array}{ll}
\ddot{u}(x, t)-u_{x x}(x, t)=0, & (x, t) \in \mathbb{R} \times(0, \infty), \\
u(x+2 \pi, t)=u(x, t), & (x, t) \in \mathbb{R} \times(0, \infty), \\
u(x, 0)=u_{0}(x), \dot{u}(x, 0)=v_{0}(x), & x \in \mathbb{R} .
\end{array}
$$

The proof of the lemma can be adapted from [15] and we omit the details. Here $\dot{H}_{\text {per }}^{\alpha}$ stands for the subspace of $\dot{H}^{\alpha}$ consisting of $2 \pi$-periodic functions.

LEMma 4.4. Let $u$ be the solution of (4.21) and $u_{h}$ its finite element approximation. Assume that, for some $\beta \geq 0$, there is a constant $C$ such that for all $u_{0} \in \dot{H}_{\mathrm{per}}^{\alpha}, v_{0} \in \dot{H}_{\mathrm{per}}^{\alpha-1}$ and $h>0$,

$$
\left\|u(t)-u_{h}(t)\right\| \leq C h^{\frac{2}{3} \beta}\left(\left\|u_{0}\right\|_{\dot{H}_{\mathrm{per}}^{\alpha}}+\left\|v_{0}\right\|_{\dot{H}_{\mathrm{per}}^{\alpha-1}}\right), \quad t \geq 0 .
$$

Then $\alpha \geq \beta$.

Remark 4.5. Error estimates of optimal order in $L_{\infty}\left([0, \infty], \dot{H}^{0}\right)$ for the finite element approximation of displacement $u=u_{1}$ and velocity $\dot{u}=u_{2}$ were first obtained in [7. However, the regularity requirement is not minimal there. This was improved in [3] and in [15] it was shown that the resulting regularity requirement is minimal, see Lemma 4.4. The error estimate (4.11) can be found in [3, while (4.9) and $(4.10)$ seem to be new. Furthermore, the proof presented here seems to be more straightforward. 
5. The finite element method for the stochastic problem. We now consider the approximation of the stochastic wave equation. The spatially discrete analogue of 2.5) is to find $X_{h}(t)=\left(u_{h, 1}(t), u_{h, 2}(t)\right) \in V_{h} \times V_{h}$ such that

$$
\mathrm{d} X_{h}(t)=A_{h} X_{h}(t) \mathrm{d} t+B \mathcal{P}_{h} \mathrm{~d} W(t), \quad t>0 ; \quad X_{h}(0)=X_{h, 0},
$$

where $A_{h}$ is defined in 4.15 . Recall that $A_{h}$ generates the $C_{0}$-semigroup $E_{h}(t)=\mathrm{e}^{t A_{h}}$ on $V_{h}$ given by (4.16), and therefore the unique mild solution of (5.1) is given by

$$
X_{h}(t)=E_{h}(t) X_{h, 0}+\int_{0}^{t} E_{h}(t-s) B \mathcal{P}_{h} \mathrm{~d} W(s), \quad t \geq 0 .
$$

Recall $\|\mid v\|\left\|_{\alpha}^{2}=\right\| v_{1}\left\|_{\alpha}^{2}+\right\| v_{2} \|_{\alpha-1}^{2}$ from (3.1).

THEOREM 5.1. Let $X_{0}=\left[u_{0}, v_{0}\right]^{T}$ and let $X=\left[u_{1}, u_{2}\right]^{T}$ and $X_{h}=\left[u_{h, 1}, u_{h, 2}\right]^{T}$ be given by (3.4) and (5.2), respectively. Then, the following estimates hold for $t \geq 0$, where $C_{t}$ is an increasing function of $t$.

If $u_{h, 0}=\mathcal{P}_{h} u_{0}, v_{h, 0}=\mathcal{P}_{h} v_{0}$, and $\beta \in[0,3]$, then

$$
\left\|u_{h, 1}(t)-u_{1}(t)\right\|_{L_{2}\left(\Omega, \dot{H}^{0}\right)} \leq C_{t} h^{\frac{2}{3} \beta}\left\{\left\|X_{0}\right\|_{L_{2}\left(\Omega, H^{\beta}\right)}+\left\|\Lambda^{\frac{1}{2}(\beta-1)} Q^{1 / 2}\right\|_{\mathrm{HS}}\right\} .
$$

If $u_{h, 0}=\mathcal{R}_{h} u_{0}, v_{h, 0}=\mathcal{P}_{h} v_{0}$, and $\beta \in[1,3]$, then

$$
\left\|u_{h, 1}(t)-u_{1}(t)\right\|_{L_{2}\left(\Omega, \dot{H}^{1}\right)} \leq C_{t} h^{\frac{1}{2}(\beta-1)}\left\{\left\|X_{0}\right\|_{L_{2}\left(\Omega, H^{\beta}\right)}+\left\|\Lambda^{\frac{1}{2}(\beta-1)} Q^{1 / 2}\right\|_{\mathrm{HS}}\right\} .
$$

If $u_{h, 0}=\mathcal{R}_{h} u_{0}, v_{h, 0}=\mathcal{P}_{h} v_{0}$, and $\beta \in[1,4]$, then

$$
\left\|u_{h, 2}(t)-u_{2}(t)\right\|_{L_{2}\left(\Omega, \dot{H}^{0}\right)} \leq C_{t} h^{\frac{2}{3}(\beta-1)}\left\{\left\|X_{0}\right\|_{L_{2}\left(\Omega, H^{\beta}\right)}+\left\|\Lambda^{\frac{1}{2}(\beta-1)} Q^{1 / 2}\right\|_{\mathrm{HS}}\right\} .
$$

The discrete initial values $\left(u_{h, 0}=\mathcal{P}_{h} u_{0}\right.$, or $u_{h, 0}=\mathcal{R}_{h} u_{0}$, and $\left.v_{h, 0}=\mathcal{P}_{h} v_{0}\right)$ and the regularity of the initial values $\left(X_{0} \in H^{\beta}\right)$ are chosen so that the corresponding rates of convergence match those of the stochastic convolution terms. Of course, other choices are possible with different convergence rates that can be derived from Theorem 4.1

Proof. We prove 5.3); the proofs of the other estimates are similar.

In addition to $F_{h}$ defined in 4.17) we introduce

$$
K_{h}(t) f=\left(\Lambda_{h}^{-1 / 2} S_{h}(t) \mathcal{P}_{h}-\Lambda^{-1 / 2} S(t)\right) f
$$

and deduce from (4.18) with $u_{0}=0$ that

$$
\left\|K_{h}(t) f\right\| \leq C_{t} h^{\frac{2}{3} \beta}\|f\|_{\beta-1} .
$$

Then we have

$$
u_{h, 1}(t)-u_{1}(t)=F_{h}(t) X_{0}+\int_{0}^{t} K_{h}(t-s) \mathrm{d} W(s) .
$$

By Itô's isometry 2.4,

$$
\begin{aligned}
\left\|u_{h, 1}(t)-u_{1}(t)\right\|_{L_{2}(\Omega, U)} & \leq\left\|F_{h}(t) X_{0}\right\|_{L_{2}(\Omega, U)}+\left\|\int_{0}^{t} K_{h}(t-s) \mathrm{d} W(s)\right\|_{L_{2}(\Omega, U)} \\
& =\left\|F_{h}(t) X_{0}\right\|_{L_{2}(\Omega, U)}+\left(\int_{0}^{t}\left\|K_{h}(s) Q^{1 / 2}\right\|_{\mathrm{HS}}^{2} \mathrm{~d} s\right)^{1 / 2} \\
& =I+I I .
\end{aligned}
$$


From 4.18) it follows that

$$
I^{2}=\mathbf{E}\left(\left\|F_{h}(t) X_{0}\right\|^{2}\right) \leq C_{t} h^{\frac{4}{3} \beta} \mathbf{E}\left(\left\|\mid X_{0}\right\|_{\beta}^{2}\right) .
$$

Recalling the definition of the Hilbert-Schmidt norm from Section 2 using an orthonormal basis $\left\{e_{k}\right\}_{k=1}^{\infty}$ in $U=\dot{H}^{0}$, we obtain

$$
I I^{2}=\sum_{k=1}^{\infty} \int_{0}^{t}\left\|K_{h}(s) Q^{1 / 2} e_{k}\right\|^{2} \mathrm{~d} s
$$

Finally, by setting $f=Q^{1 / 2} e_{k}$ in (5.6), we conclude that

$$
I I^{2} \leq C_{t} t h^{\frac{4}{3} \beta} \sum_{k=1}^{\infty}\left\|Q^{1 / 2} e_{k}\right\|_{\beta-1}^{2}=C_{t} h^{\frac{4}{3} \beta}\left\|\Lambda^{(\beta-1) / 2} Q^{1 / 2}\right\|_{\mathrm{HS}}^{2},
$$

which completes the proof of 5.3 .

Remark 5.2. Consider the one dimensional case with space-time white noise; that is, $d=1$ and $Q=I$. Then $\beta<1 / 2$ (see Remark 3.2) and the convergence rate in (5.3) is $O\left(h^{\alpha}\right), \alpha<1 / 3$. This is in agreement with [14], while $O\left(h^{1 / 2}\right)$ was shown for the leap-frog scheme in 20 . The reason why a higher rate of convergence is obtained in [20] is that the Green's functions of the continuous and the discrete equations coincide at the mesh points. Another example of a numerical scheme where this happens is Galerkin's method for the periodic problem in Remark 4.3 with

$$
V_{h}=\operatorname{span}\left\{\mathrm{e}^{i n x}:|n| \leq 1 / h\right\},
$$

see [15, Remark 2]. Then instead of 4.18) we would have

$$
\left\|F_{h}(t) X_{0}\right\| \leq C h^{\beta}|| X_{0} \|_{\beta}, \quad t \geq 0, \quad \beta \in[0,2],
$$

and, under the assumptions of 5.3 ,

$$
\left\|u_{h, 1}(t)-u_{1}(t)\right\|_{L_{2}(\Omega, U)} \leq C h^{\beta}\left\{\left\|X_{0}\right\|_{L_{2}\left(\Omega, H^{\beta}\right)}+\left\|\Lambda^{(\beta-1) / 2} Q^{1 / 2}\right\|_{\mathrm{HS}}\right\} .
$$

This yields the order $O\left(h^{\alpha}\right), \alpha<1 / 2$, for $Q=I$.

The error estimates in Theorem 4.1 and therefore in Corollary 4.2 and Theorem 5.1. can be extended to higher order finite element methods. The reason is that the error estimates for the elliptic and the orthogonal projections in 4.2 and $(4.3)$, respectively, as well as the stability inequality (4.8) hold for higher order finite element spaces $V_{h}$ consisting of continuous piecewise polynomials of order at most $k \geq 1$. This means that in case of highly correlated noise, one might expect higher order of strong convergence when using a higher order finite element method. In this case the counterpart of Theorem 5.1 reads as follows.

THEOREM 5.3. Let $X_{0}=\left[u_{0}, v_{0}\right]^{T}$ and let $X=\left[u_{1}, u_{2}\right]^{T}$ and $X_{h}=\left[u_{h, 1}, u_{h, 2}\right]^{T}$ be given by (3.4) and (5.2), respectively, where the finite element spaces $V_{h}$ consist of continuous piecewise polynomials of order at most $k \geq 1$. Then, the following estimates hold for $t \geq 0$, where $C_{t}$ is an increasing function of $t$.

If $u_{h, 0}=\mathcal{P}_{h} u_{0}, v_{h, 0}=\mathcal{P}_{h} v_{0}$, and $\beta \in[0, k+2]$, then

$$
\left\|u_{h, 1}(t)-u_{1}(t)\right\|_{L_{2}\left(\Omega, \dot{H}^{0}\right)} \leq C_{t} h^{\frac{k+1}{k+2} \beta}\left\{\left\|X_{0}\right\|_{L_{2}\left(\Omega, H^{\beta}\right)}+\left\|\Lambda^{\frac{1}{2}(\beta-1)} Q^{1 / 2}\right\|_{\mathrm{HS}}\right\} .
$$


If $u_{h, 0}=\mathcal{R}_{h} u_{0}, v_{h, 0}=\mathcal{P}_{h} v_{0}$, and $\beta \in[1, k+2]$, then

$$
\left\|u_{h, 1}(t)-u_{1}(t)\right\|_{L_{2}\left(\Omega, \dot{H}^{1}\right)} \leq C_{t} h^{\frac{k}{k+1}(\beta-1)}\left\{\left\|X_{0}\right\|_{L_{2}\left(\Omega, H^{\beta}\right)}+\left\|\Lambda^{\frac{1}{2}(\beta-1)} Q^{1 / 2}\right\|_{\mathrm{HS}}\right\} .
$$

If $u_{h, 0}=\mathcal{R}_{h} u_{0}, v_{h, 0}=\mathcal{P}_{h} v_{0}$, and $\beta \in[1, k+3]$, then

$$
\left\|u_{h, 2}(t)-u_{2}(t)\right\|_{L_{2}\left(\Omega, \dot{H}^{0}\right)} \leq C_{t} h^{\frac{k+1}{k+2}(\beta-1)}\left\{\left\|X_{0}\right\|_{L_{2}\left(\Omega, H^{\beta}\right)}+\left\|\Lambda^{\frac{1}{2}(\beta-1)} Q^{1 / 2}\right\|_{\mathrm{HS}}\right\} .
$$

6. Numerical experiments. In this section we demonstrate the order of strong convergence of the finite element method for the linear stochastic wave equation LSWE (1.1) by numerical examples. To this end, the backward Euler method is used for time stepping and some computational analysis on the approximation of the stochastic convolution is reviewed, see 22 .

6.1. Computational analysis. First recall the matrix form of (5.1),

$$
\left[\begin{array}{l}
\mathrm{d} u_{h, 1}(t) \\
\mathrm{d} u_{h, 2}(t)
\end{array}\right]=\left[\begin{array}{cc}
0 & I \\
-\Lambda_{h} & 0
\end{array}\right]\left[\begin{array}{l}
u_{h, 1}(t) \\
u_{h, 2}(t)
\end{array}\right] \mathrm{d} t+\left[\begin{array}{c}
0 \\
\mathcal{P}_{h} \mathrm{~d} W(t)
\end{array}\right]
$$

Let $0=t_{0}<t_{1}<\cdots<t_{N_{t}}=T_{N}$, be a uniform partition of the time interval $\left[0, T_{N}\right]$ with time step $k=1 / N_{t}$ and time subintervals $I_{n}=\left(t_{n-1}, t_{n}\right), n=1,2, \cdots, N_{t}$. Then the backward Euler method is formulated as, for $n=1,2, \cdots, N_{t}$,

$$
\left[\begin{array}{l}
U_{1}^{n} \\
U_{2}^{n}
\end{array}\right]-\left[\begin{array}{l}
U_{1}^{n-1} \\
U_{2}^{n-1}
\end{array}\right]=\left[\begin{array}{cc}
0 & k I \\
-k \Lambda_{h} & 0
\end{array}\right]\left[\begin{array}{c}
U_{1}^{n} \\
U_{2}^{n}
\end{array}\right]+\left[\begin{array}{c}
0 \\
\mathcal{P}_{h} \Delta W^{n}
\end{array}\right]
$$

Here $U_{i}^{n} \in V_{h}$ is an approximation of $u_{i}\left(\cdot, t_{n}\right), i=1,2$. We multiply 6.1 by

$$
\left[\begin{array}{cc}
\Lambda_{h} & 0 \\
0 & I
\end{array}\right]
$$

to take advantage of the resulting skew-symmetric structure and rearrange, to obtain, for $n=1,2, \cdots, N_{t}$,

$$
\left[\begin{array}{cc}
\Lambda_{h} & -k \Lambda_{h} \\
k \Lambda_{h} & I
\end{array}\right]\left[\begin{array}{c}
U_{1}^{n} \\
U_{2}^{n}
\end{array}\right]=\left[\begin{array}{cc}
\Lambda_{h} & 0 \\
0 & I
\end{array}\right]\left[\begin{array}{l}
U_{1}^{n-1} \\
U_{2}^{n-1}
\end{array}\right]+\left[\begin{array}{c}
0 \\
\mathcal{P}_{h} \Delta W^{n}
\end{array}\right]
$$

For some other ways of approximating the noise and the stochastic integrals we refer to, for example, [2] and [6].

Recalling the Fourier expansion 2.2 of $W$, we have, for all $\chi \in V_{h}$,

$$
\left(\mathcal{P}_{h} \Delta W^{n}, \chi\right)=\sum_{j=1}^{\infty} \gamma_{j}^{1 / 2} \Delta \beta_{j}^{n}\left(e_{j}, \chi\right) \approx \sum_{j=1}^{J} \gamma_{j}^{1 / 2} \Delta \beta_{j}^{n}\left(e_{j}, \chi\right),
$$

where we truncated the sum to $J$ terms. Recall that $\left\{\beta_{j}(t)\right\}_{j=1}^{J}$ are mutually independent standard real-valued Brownian motions, and that the increments in 6.3 are

$$
\Delta \beta_{j}^{n}=\beta_{j}\left(t_{n}\right)-\beta_{j}\left(t_{n-1}\right) \sim \sqrt{k} \mathcal{N}(0,1),
$$

that is, real-valued Gaussian random variables with 0 mean and variance $k$. We also note that $\gamma_{j}=1$ for white noise. 
Recalling the semidiscrete solution $u_{h}$ from 5.2 , we denote by $u_{h}^{J}$ the semidiscrete solution obtained by using the truncated noise; that is,

$$
u_{h}^{J}(t)=E_{h}(t) X_{0, h}+\sum_{j=1}^{J} \gamma_{j}^{1 / 2} \int_{0}^{t} E_{h}(t-s) B \mathcal{P}_{h} e_{j} \mathrm{~d} \beta_{j}(s) .
$$

The following lemma shows, that under some assumptions on the triangulation and the covariance operator $Q$, it is enough to take $J \geq N_{h}$ with $N_{h}=\operatorname{dim}\left(V_{h}\right)$ in order to preserve the order of the finite element method.

Lemma 6.1. Let $u_{h}^{J}$ and $u_{h}$ be defined by (6.4) and (5.2), respectively. Assume that $\Lambda$ and $Q$ have a common orthonormal basis of eigenfunctions $\left\{e_{j}\right\}_{j=1}^{\infty}$ and that $V_{h}$, with dimension $N_{h}$, is defined on a family of quasi-uniform triangulations $\left\{\mathcal{T}_{h}\right\}$ of $\mathcal{D}$. Then for $J \geq N_{h}$ the following estimates hold, where $C_{t}$ is an increasing function.

If $\left\|\Lambda^{(\beta-1) / 2} Q^{1 / 2}\right\|_{\mathrm{HS}}<\infty$ for some $\beta \in[0,3]$, then,

$$
\left\|u_{h, 1}^{J}(t)-u_{h, 1}(t)\right\|_{L_{2}\left(\Omega, \dot{H}^{0}\right)} \leq C_{t} h^{\frac{2}{3} \beta}\left\|\Lambda^{(\beta-1) / 2} Q^{1 / 2}\right\|_{\mathrm{HS}} .
$$

If $\left\|\Lambda^{(\beta-1) / 2} Q^{1 / 2}\right\|_{\mathrm{HS}}<\infty$ for some $\beta \in[1,3]$, then,

$$
\left\|u_{h, 1}^{J}(t)-u_{h, 1}(t)\right\|_{L_{2}\left(\Omega, \dot{H}^{1}\right)} \leq C_{t} h^{\frac{1}{2}(\beta-1)}\left\|\Lambda^{(\beta-1) / 2} Q^{1 / 2}\right\|_{\mathrm{HS}} .
$$

If $\left\|\Lambda^{(\beta-1) / 2} Q^{1 / 2}\right\|_{\mathrm{HS}}<\infty$ for some $\beta \in[1,4]$, then,

$$
\left\|u_{h, 2}^{J}(t)-u_{h, 2}(t)\right\|_{L_{2}\left(\Omega, \dot{H}^{0}\right)} \leq C_{t} h^{\frac{2}{3}(\beta-1)}\left\|\Lambda^{(\beta-1) / 2} Q^{1 / 2}\right\|_{\mathrm{HS}} .
$$

Proof. We prove the second estimate; the others are proved similarly. From 5.2 and (6.4) it follows that

$$
u_{h, 1}^{J}(t)-u_{h, 1}(t)=\sum_{j=J+1}^{\infty} \gamma_{j}^{1 / 2} \int_{0}^{t} \Lambda_{h}^{-1 / 2} S_{h}(t-s) \mathcal{P}_{h} e_{j} \mathrm{~d} \beta_{j}(s) .
$$

By Itô's isometry (2.4), the independence of $\beta_{j}$ 's, and recalling the error operator from (5.5), we have

$$
\begin{aligned}
& \left\|u_{h, 1}^{J}(t)-u_{h, 1}(t)\right\|_{L_{2}\left(\Omega, \dot{H}^{1}\right)}^{2}=\sum_{j=J+1}^{\infty} \gamma_{j} \int_{0}^{t}\left\|\Lambda_{h}^{-1 / 2} S_{h}(s) \mathcal{P}_{h} e_{j}\right\|_{1}^{2} \mathrm{~d} s \\
& \quad \leq 2 \sum_{j=J+1}^{\infty} \gamma_{j} \int_{0}^{t}\left\|\Lambda^{-1 / 2} S(s) e_{j}\right\|_{1}^{2} \mathrm{~d} s+2 \sum_{j=J+1}^{\infty} \gamma_{j} \int_{0}^{t}\left\|K_{h}(s) e_{j}\right\|_{1}^{2} \mathrm{~d} s=I+I I .
\end{aligned}
$$

Let $\lambda_{j}$ denote the eigenvalues of $\Lambda$ corresponding to $e_{j}$. Then

$$
\left\|\Lambda^{-1 / 2} \sin \left(s \Lambda^{1 / 2}\right) e_{j}\right\|_{1}^{2}=\sin ^{2}\left(s \lambda_{j}^{1 / 2}\right) .
$$

Thus,

$$
\begin{aligned}
I & =2 \sum_{j=J+1}^{\infty} \gamma_{j} \int_{0}^{t}\left\|\Lambda^{-1 / 2} \sin \left(s \Lambda^{1 / 2}\right) e_{j}\right\|_{1}^{2} \mathrm{~d} s \\
& =2 \sum_{j=J+1}^{\infty} \gamma_{j} \int_{0}^{t} \sin ^{2}\left(s \lambda_{j}^{1 / 2}\right) \mathrm{d} s \leq 2 t \sum_{j=J+1}^{\infty} \gamma_{j}=2 t \sum_{j=J+1}^{\infty} \lambda_{j}^{-(\beta-1)}\left(\lambda_{j}^{\beta-1} \gamma_{j}\right) \\
& \leq 2 t \lambda_{J+1}^{-(\beta-1)} \sum_{j=J+1}^{\infty} \lambda_{j}^{\beta-1} \gamma_{j} \leq 2 t \lambda_{J+1}^{-(\beta-1)}\left\|\Lambda^{(\beta-1) / 2} Q^{1 / 2}\right\|_{\mathrm{HS}}^{2} .
\end{aligned}
$$


For $I I$, by 4.19 with $u_{0}=0, v_{0}=e_{j}$, we have

$$
\begin{aligned}
I I & \leq C_{t} h^{\beta-1} \sum_{j=J+1}^{\infty} \gamma_{j} \int_{0}^{t}\left\|e_{j}\right\|_{\beta-1}^{2} \mathrm{~d} s \\
& =C_{t} h^{\beta-1} \sum_{j=J+1}^{\infty} \gamma_{j}\left\|e_{j}\right\|_{\beta-1}^{2} \leq C_{t} h^{\beta-1}\left\|\Lambda^{(\beta-1) / 2} Q^{1 / 2}\right\|_{\mathrm{HS}}^{2} .
\end{aligned}
$$

Hence the proof is completed by the fact that, for a quasi-uniform family of triangulations, we have $N_{h} \approx h^{-d}$ and therefore, since $\lambda_{j} \approx j^{2 / d}$,

$$
\lambda_{J+1}^{-1} \leq C J^{-2 / d} \leq C N_{h}^{-2 / d} \leq C h^{2} .
$$

This completes the proof.

Remark 6.2. In practice $Q$ and $\Lambda$ do not have a common orthonormal basis of eigenfunctions and the eigenfunctions of $Q$ are not known explicitly. In this case, one has to solve the eigenvalue problem $Q u=\lambda u$ on $S_{h}$ in order to represent $\mathcal{P}_{h} W$. Computationally this could be very expensive if $Q$ is given by an integral operator. However, if the kernel is smooth then this can be done more efficiently, see [17]. Furthermore, similarly to the parabolic case [11, it is enough to keep $J<N_{h}$ terms, for suitable $J$ depending on the kernel, in the expansion of $\mathcal{P}_{h} W$.

6.2. Numerical example. For the numerical experiments, we consider the LSWE in one spatial dimension,

$$
\begin{array}{ll}
\mathrm{d} \dot{u}-\Delta u \mathrm{~d} t=\mathrm{d} W, & (x, t) \in(0,1) \times(0,1), \\
u(0, t)=u(1, t)=0, & t \in(0,1), \\
u(x, 0)=\cos (\pi(x-1 / 2)), u_{t}(x, 0)=0, & x \in(0,1) .
\end{array}
$$

Clearly, there is no exact solution available. Therefore we take the exact solution to be a finite element approximation on a very fine mesh with mesh size $h_{\text {exact }}$ to approximate $u=u(x, 1)$, using the backward Euler method 6.2 for time stepping with a small fixed time step $k$. We note that we choose the time step $k$ according to $k \leq h^{2}$, since the rate of convergence of the fully discrete 6.2 for the deterministic problem is $O\left(k+h^{2}\right)$.

Applying the time stepping 6.2 to 6.5 we obtain the discrete system

$$
\Sigma X^{n}=\Xi X^{n-1}+b
$$

where $b=\left[0, b_{2}\right]^{T}$ and $b_{2}$ is computed using (6.3). We note that for the deterministic problem $b=0$, the expected rate of convergence in the $L_{2}$-norm for both the displacement $u=u_{1}$ and the velocity $\dot{u}=u_{2}$ is 2 by 4.11) and 4.10, respectively, see Figure 6.1.

If $\left\{\lambda_{j}\right\}_{j=1}^{\infty}$ are the eigenvalues of $\Lambda$, and we set $Q=\Lambda^{-s}, s \in \mathbb{R}$, then

$$
\left\|\Lambda^{(\beta-1) / 2} Q^{1 / 2}\right\|_{\mathrm{HS}}^{2}=\left\|\Lambda^{(\beta-s-1) / 2}\right\|_{\mathrm{HS}}^{2}=\sum_{j=1}^{\infty} \lambda_{j}^{\beta-s-1} \approx \sum_{j=1}^{\infty} j^{\frac{2}{d}(\beta-s-1)},
$$

which is finite if and only if $\beta<1+s-d / 2$ with $d$ being the dimension of the domain $\mathcal{D}$. In our example 6.5), where $d=1$, we consider two different choices for the noise. 
First, we consider space-time white noise corresponding to $s=0$ and hence $\beta<1 / 2$ and then a correlated noise corresponding $s=1$ and hence $\beta<3 / 2$. Thus, in the case of space-time white noise, we do not expect convergence for the finite element approximation of velocity $u_{h, 2}$ by (5.4), but we expect the rate of convergence to be $1 / 3$ for displacement $u_{h, 1}$ by $(5.3)$. These are confirmed by Figure 6.2 . In the second case, the expected rate of strong convergence is 1 and $1 / 3$ for displacement and velocity by (5.3) and (5.4), respectively, as Figure 6.3 also confirms. We note that we have used a uniform spatial mesh and therefore with $Q=\Lambda^{-1}$, the assumptions of Lemma 6.1 are fulfilled.

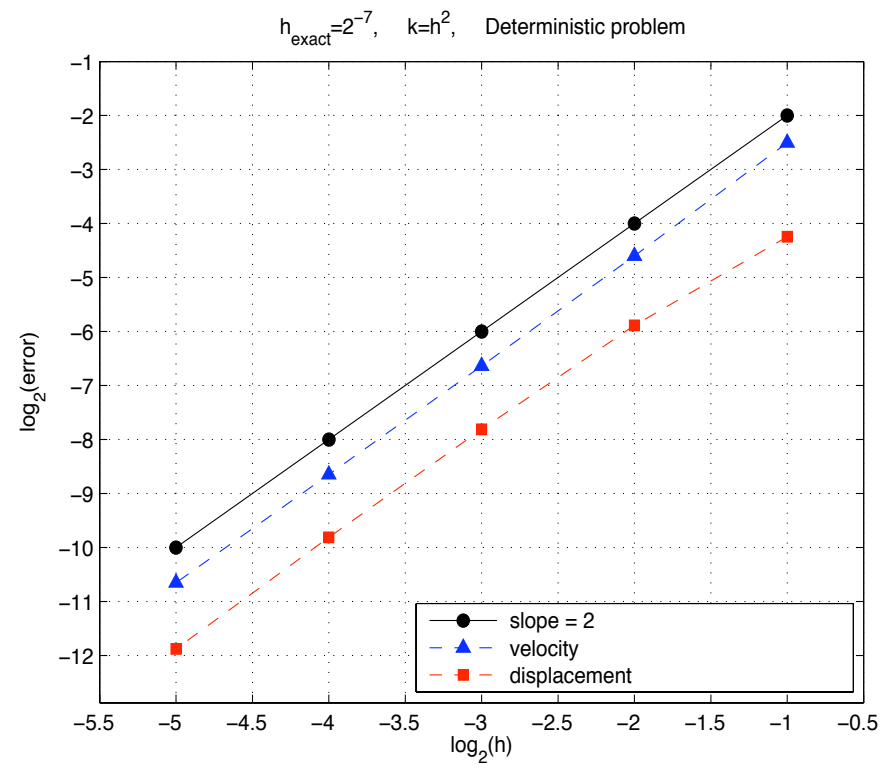

FIG. 6.1. Deterministic problem: the order of strong convergence in the $L_{2}$-norm is 2 for both the displacement $u$ (dashed-square) and the velocity $\dot{u}$ (dashed-triangle).

\section{REFERENCES}

[1] E. J. Allen, Modeling with Itô Stochastic Differential Equations, vol. 22 of Mathematical Modelling: Theory and Applications, Springer, Dordrecht, 2007.

[2] E. J. Allen, S. J. Novosel, And Z. Zhang, Finite element and difference approximation of some linear stochastic partial differential equations, Stochastics Stochastics Rep., 64 (1998), pp. 117-142.

[3] G. A. BAKER, Error estimates for finite element methods for second order hyperbolic equations, SIAM J. Numer. Anal., 13 (1976), pp. 564-576.

[4] P. CHow, Stochastic Partial Differential Equations, Chapman \& Hall/CRC, Boca Raton, FL, 2007.

[5] G. Da Prato and J. Zabczyk, Stochastic Equations in Infinite Dimensions, Cambridge University Press, Cambridge, 1992.

[6] Q. Du AND T. Zhang, Numerical approximation of some linear stochastic partial differential equations driven by special additive noises, SIAM J. Numer. Anal., 40 (2002), pp. 14211445 (electronic).

[7] T. Dupont, $L^{2}$-estimates for Galerkin methods for second order hyperbolic equations, SIAM J. Numer. Anal., 10 (1973), pp. 880-889.

[8] M. Geissert, M. Kovács, And S. Larsson, Rate of weak convergence of the finite element method for the stochastic heat equation with additive noise, BIT, 49 (2009), pp. 343-356. 


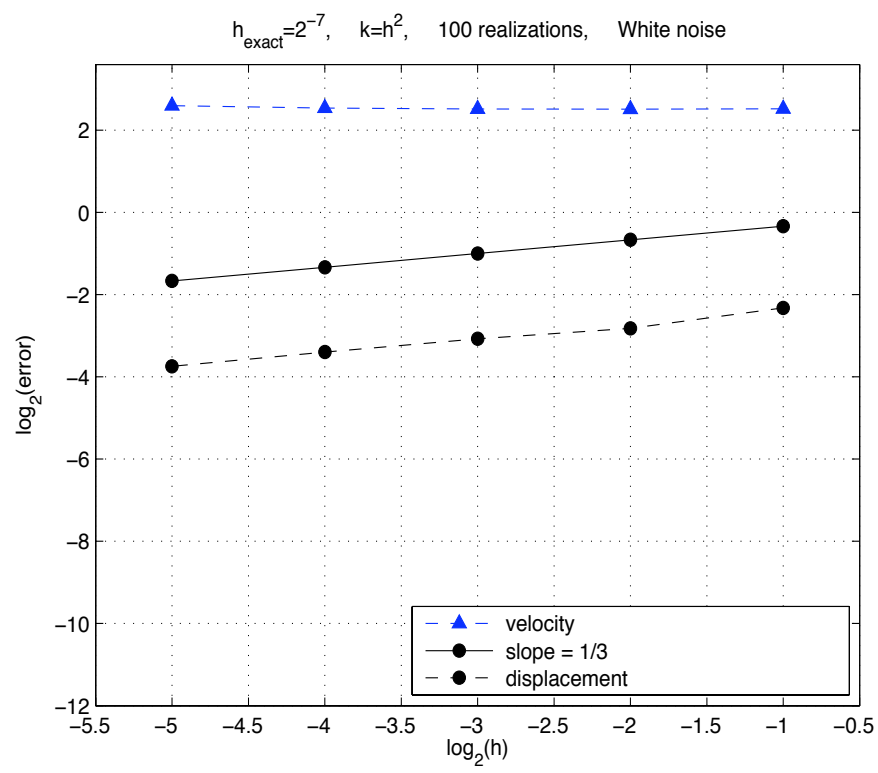

FIG. 6.2. LSWE with white noise: the order of strong convergence in the $L_{2}$-norm is $1 / 3$ for the displacement $u$ (dashed-circle); but there is no convergence for the velocity $\dot{u}$ (dashed-triangle).

[9] I. GYÖNGY, Approximations of stochastic partial differential equations, in Stochastic partial differential equations and applications (Trento, 2002), vol. 227 of Lecture Notes in Pure and Appl. Math., Dekker, New York, 2002, pp. 287-307.

[10] M. Kovács, S. Larsson, ANd F. Lindgren, Weak convergence of finite element approximations of linear stochastic evolution equations with additive noise, (2009). Preprint.

[11] — Strong convergence of the finite element method with truncated noise for semilinear parabolic stochastic equations with additive noise, Numer. Algorithms, 53 (2010), pp. 309320.

[12] A. Martin, S. M. Prigarin, And G. Winkler, Exact and fast numerical algorithms for the stochastic wave equation, Int. J. Comput. Math., 80 (2003), pp. 1535-1541.

[13] C. Prévôt And M. Röckner, A Concise Course on Stochastic Partial Differential Equations, vol. 1905 of Lecture Notes in Mathematics, Springer, Berlin, 2007.

[14] L. Quer-Sardanyons and M. SAnZ-Solé, Space semi-discretisations for a stochastic wave equation, Potential Anal., 24 (2006), pp. 303-332.

[15] J. RAUCH, On convergence of the finite element method for the wave equation, SIAM J. Numer. Anal., 22 (1985), pp. 245-249.

[16] C. Rотн, Weak approximations of solutions of a first order hyperbolic stochastic partial differential equation, Monte Carlo Methods Appl., 13 (2007), pp. 117-133.

[17] C. SCHWAB AND R. A. TOdoR, Karhunen-Loève approximation of random fields by generalized fast multipole methods, J. Comput. Phys., 217 (2006), pp. 100-122.

[18] V. Tноме́е, Galerkin Finite Element Methods for Parabolic Problems, Springer-Verlag, Berlin, second ed., 2006.

[19] J. B. WALSH, An introduction to stochastic partial differential equations, in École d'été de probabilités de Saint-Flour, XIV-1984, vol. 1180 of Lecture Notes in Math., Springer, Berlin, 1986, pp. 265-439.

[20] - On numerical solutions of the stochastic wave equation, Illinois J. Math., 50 (2006), pp. 991-1018 (electronic).

[21] Y. YAN, Semidiscrete Galerkin approximation for a linear stochastic parabolic partial differential equation driven by an additive noise, BIT, 44 (2004), pp. 829-847.

[22] — Galerkin finite element methods for stochastic parabolic partial differential equations, SIAM J. Numer. Anal., 43 (2005), pp. 1363-1384 (electronic). 


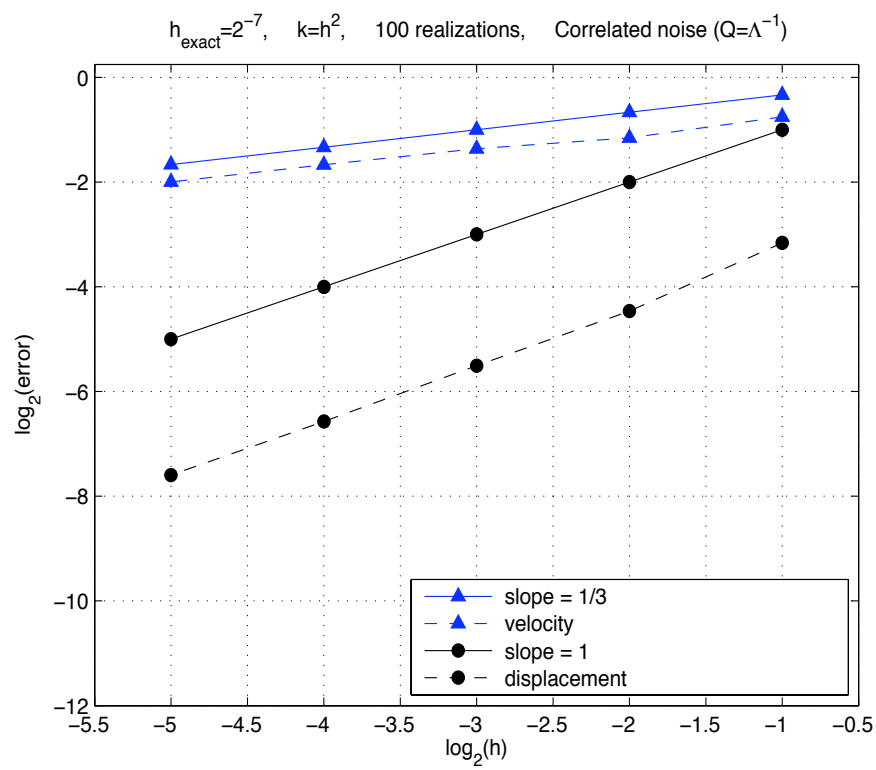

FIG. 6.3. LSWE with correlated noise $Q=\Lambda^{-1}$ : the order of strong convergence in the $L_{2}$-norm is 1 for the displacement $u$ (dashed-circle), and $1 / 3$ for the velocity $\dot{u}$ (dashed-triangle). 